\section{Petrología de morteros en Arqueología. Catedral de Santa María de Vitoria-Gasteiz}

\author{
BLANCA GUARÁS
}

Geóloga

\section{INTRODUCCIÓN}

La Arqueología, en su intento de avanzar en el conocimiento de la cultura material de las diferentes civilizaciones, ha necesitado de una serie de ciencias aplicadas con el fin de dar respuesta a los diferentes problemas que se le plantean. La Petrología, como ciencia que estudia el material rocoso, se ha convertido recientemente, en una herramienta de gran interés para el arqueólogo. Puede ser estudiado por esta ciencia todo elemento arqueológico que esté constituido por material rocoso, ya sea natural o elaborado (morteros, cerámica, etc).

Un mortero, o argamasa, es una mezcla de arena ligada por un aglomerante de cal, yeso ó cemento, y que sirve para dar trabazón a los elementos de una construcción arquitectónica, así como para cimentar, rellenar, revestir y reparar. Por tanto, el estudio petrológico de morteros en un conjunto arquitectónico antiguo nos permite:

- Conocer los materiales utilizados en las distintas etapas de construcción y restauraciones recientes.

- Evaluar el estado de conservación de los morteros.

-Correlacionar las características petrológicas de los distintos morteros con su función constructiva.

-Deducir la procedencia del material y establecer posibles áreas fuente, a partir de datos de geología regional.

- Reconocer morteros «idénticos», dato que nos indica una misma fase constructiva.

Finalmente, el estudio de morteros antiguos contribuye a la investigación de la evolución espacio-temporal del conjunto arquitectónico, y representa una base de conocimiento para los trabajos de conservación y restauración del patrimonio arquitectónico.

\section{METODOLOGÍA}

El método analítico está basado, fundamentalmente, en el estudio petrográfico con microscopio de luz polarizada de una sección delgada del mortero.

El primer paso a realizar es el muestreo, de cuya rigurosidad y planteamiento dependerá, en gran parte, el éxito del trabajo. El criterio de selección de las muestras debe estar en función de los objetivos planteados. La recogida de muestras se realiza, principalmente, in situ, aunque en ocasiones hay que recurrir a sondeos de testigo continuo para los puntos inaccesibles de interés. En la toma de muestras es necesario recoger una cantidad de mortero lo más representativa posible, y el tamaño depende de la granulometría y homogeneidad del mortero. En el caso de que detectemos la existencia de más de una capa de mortero en el mismo punto, recogemos la muestra de tal manera que constituyan un único bloque. 


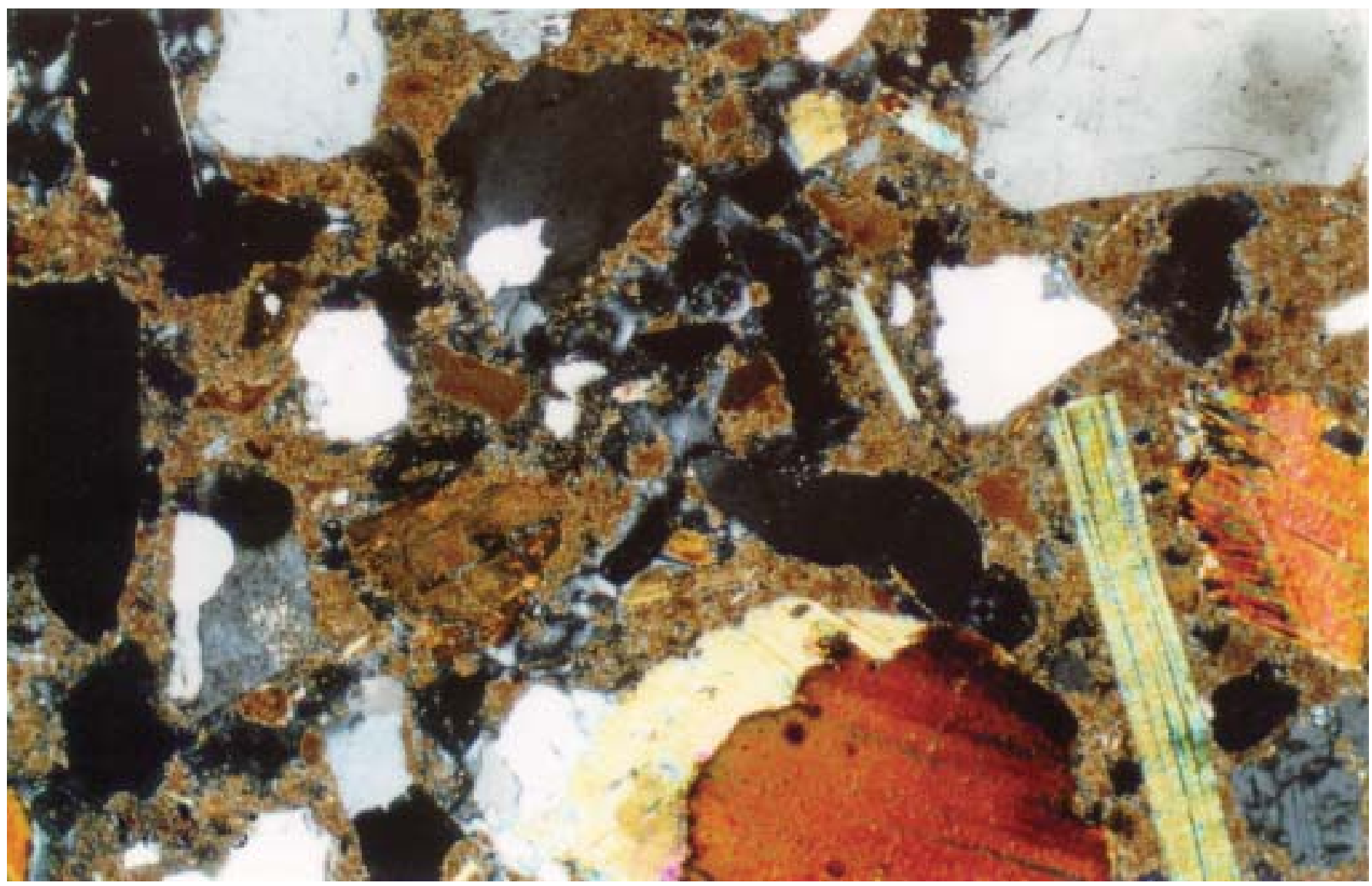

Fig. 1. Fotomicrografía de un mortero de cal con áridos de origen granítico. Bóveda de la Iglesia de Santa Comba de Bande (Orense)

Una vez obtenida la muestra, se realiza su consolidación con resina en el laboratorio, de manera que forme un bloque lo suficientemente consistente para poder realizar un corte orientado y elaborar la lámina delgada (30 micras de espesor).

La caracterización petrográfica atiende a criterios composicionales, texturales y granulométricos (fig. 1). Tras un tratamiento estadístico de los datos, se sintetizan en una ficha técnica para cada muestra, junto con otros de tipo geológico y arqueológico.

En ocasiones, ha sido necesaria la utilización de técnicas complementarias, como la difracción de rayos $\mathrm{X}$, para identificar especies minerales imposibles de detectar con microscopio de polarización.

Finalmente, un estudio comparativo de los distintos morteros nos permite establecer una serie de consideraciones y valoraciones, para poder definir unas tipologías de morteros y realizar unas conclusiones generales.

Un dato de gran interés para los arqueólogos es el poder relacionar muestras «idénticas» en distintos puntos del conjunto arquitectónico. El estudio está basado en que morteros con las mismas características petrológicas y petrográficas (morteros «idénticos») pertenecen a una misma fase cons- tructiva, ya que resulta prácticamente imposible elaborar morteros «idénticos» con una diferencia temporal de decenas o centenas de años. Con tal diferencia cronológica pueden existir morteros con características petrológicas similares, debido a la coincidencia del área fuente del material o a técnicas de elaboración semejantes, pero con características petrográficas (textura del aglomerante, proporción composicional, forma y tamaño de los áridos) ligeramente diferentes. Sin embargo, cuando encontramos morteros similares, pero no «idénticos», con una misma función constructiva en una misma unidad arquitectónica/arqueológica, no siempre significa que pertenezcan a etapas distintas, ya que un ligero cambio en el punto de extracción de la arena o en la preparación de la cal, podría modificar las características petrográficas del mortero, caso que puede darse en el transcurso de una obra. También existen diferencias en la calidad de la cal utilizada en cada etapa constructiva. Siguiendo este razonamiento, morteros «diferentes» con una misma función constructiva, corresponden a distinto momento de intervención.

\section{LA CATEDRAL DE SANTA MARIA DE VITORIA-GASTEIZ} En los trabajos de investigación enmarcados dentro del Plan Director para la restauración de la Catedral de Santa 
María de Vitoria-Gasteiz, se realizó un estudio petrológico de morteros. Este trabajo se ha ido ampliando en función de las necesidades surgidas a lo largo de las tareas de excavación arqueológica, con el fin de poder realizar una lectura estratigráfica completa del conjunto de la Catedral.

Se tomaron muestras de cimentaciones, rellenos, juntas de sillar y mampostería, enlucidos y reparaciones, distribuidos en las cuatro plantas del edificio (excavación, planta baja, paso de ronda y triforio), en el pórtico y la torre.

Una síntesis de los resultados obtenidos es la siguiente:

1. Los morteros más frecuentes son los que incorporan cal como único aglomerante; los morteros de yeso aparecen en algunos enlucidos y los morteros de cal y cemento (morteros bastardos) en reparaciones tardías.

2. La composición litológica de los áridos principales es: caliza (calcarenitas, calizas bioclásticas, calizas fosilíferas, lumaquelas, calizas dolomíticas y dolomías), cuarzo y arenisca (arenisca cuarcítica tipo Elguea). El área fuente de estos materiales está en arenas de carácter comarcal. Los áridos accesorios son fragmentos de fósiles (foraminíferos, equinodermos, etc), de cerámica y minerales opacos.
3. Los morteros utilizados en la construcción de la Catedral de Santa María presentan una variedad petrográfica atendiendo más a criterios de uso que a criterios cronológicos.

4. En cimentaciones y rellenos predominan morteros magros de cal de alta granulometría (tipos I y II); en juntas de sillar y mampostería predominan morteros intermedios y grasos de cal de granulometría media (tipos $\mathrm{V}$ y VI) y en enlucidos, morteros de cal grasos de granulometría fina (tipo VIII) y en ocasiones morteros con yeso (tipos IX y X). Para las reparaciones se utilizan diversos morteros de cal, dependiendo de su función, y morteros de cal y cemento (tipo XI) (fig. 2).

5. En las primeras fases constructivas los morteros utilizados para dar trabazón a los sillares y mampuestos son más magros que los utilizados en fases constructivas posteriores.

6. Además, hemos podido relacionar morteros «idénticos», indicativos de etapas constructivas determinadas, dato que contribuye a la lectura estratigráfica y a la investigación de la evolución espacio-temporal del conjunto de la Catedral de Santa María.

\begin{tabular}{|c|c|c|c|}
\hline \multicolumn{4}{|c|}{ MORTEROS DE CAL } \\
\hline & $\mathrm{AG}=40 \%$ & $40 \%<\mathrm{AG}<60 \%$ & $A G>-60 \%$ \\
\hline Grano $>1 \mathrm{~cm}$ & I & III & III \\
\hline $1 \mathrm{~cm}>$ Grano $>1 \mathrm{~mm}$ & IV & $\mathrm{V}$ & VI \\
\hline Grangelmam & & VII & VIII \\
\hline \multicolumn{3}{|c|}{ MORTEROS DE CAL Y YESO } & IX \\
\hline \multicolumn{3}{|c|}{ MORTEROS DE YESO } & $\mathrm{X}$ \\
\hline \multicolumn{3}{|c|}{ MORTEROS DE CAL Y CEMENTO } & $\mathrm{XI}$ \\
\hline \multicolumn{3}{|l|}{ HORMIGON } & XII \\
\hline
\end{tabular}

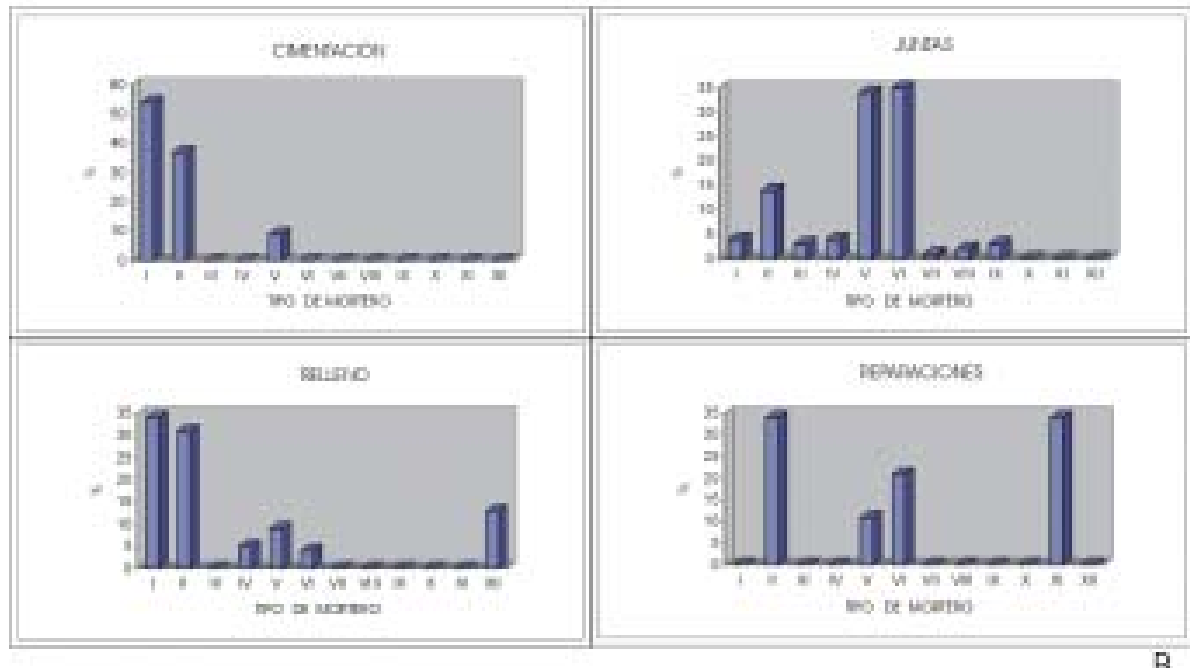

Fig. 2. Ejemplo de la Catedral de Santa María de Vitoria-Gasteiz. A: Tipos de morteros observados, en función del carácter del aglomerante y de la proporción y granulometría de los áridos. B: Correlación entre los distintos tipos de mortero y su utilización 